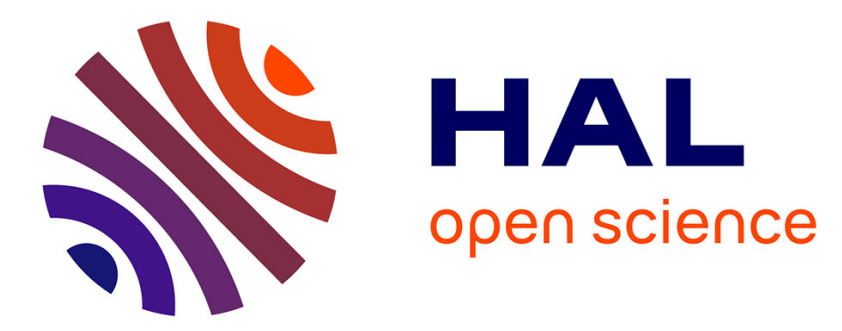

\title{
TLP-based Human Metal Model stress generator and analysis method of ESD generators
}

Rémi Bèges, Fabrice Caignet, Patrice Besse, Jean-Philippe Laine, Alain Salles, Nicolas Mauran, Nicolas Nolhier, Marise Bafleur

\section{- To cite this version:}

Rémi Bèges, Fabrice Caignet, Patrice Besse, Jean-Philippe Laine, Alain Salles, et al.. TLP-based Human Metal Model stress generator and analysis method of ESD generators. Electrical Overstress / Electrostatic Discharge Symposium (EOS/ESD 2015), Sep 2015, Reno, United States. 10.1109/EOSESD.2015.7314777 . hal-01239451

\section{HAL Id: hal-01239451 https://hal.science/hal-01239451}

Submitted on 7 Dec 2015

HAL is a multi-disciplinary open access archive for the deposit and dissemination of scientific research documents, whether they are published or not. The documents may come from teaching and research institutions in France or abroad, or from public or private research centers.
L'archive ouverte pluridisciplinaire HAL, est destinée au dépôt et à la diffusion de documents scientifiques de niveau recherche, publiés ou non, émanant des établissements d'enseignement et de recherche français ou étrangers, des laboratoires publics ou privés. 


\title{
TLP-based Human Metal Model stress generator and analysis method of ESD generators
}

\author{
Rémi Bèges ${ }^{1,2}$, Fabrice Caignet ${ }^{2,3}$, Patrice Besse ${ }^{1}$, Jean-Philippe Laine ${ }^{1}$, Alain Salles ${ }^{1}$, \\ Nicolas Mauran ${ }^{2}$, Nicolas Nolhier ${ }^{2,3}$, Marise Bafleur ${ }^{2}$ \\ (1) Freescale Semiconducteurs France SAS 2, 134 avenue du Général Eisenhower, 31650 Toulouse, France \\ e-mail: remi.beges@freescale.com \\ (2) CNRS, LAAS, 7 avenue du Colonel Roche, F-31400 Toulouse, France
}

(3) Univ. de Toulouse, UPS, LAAS, F-31400, Toulouse, France

50 Words Abstract - A new setup for generating a Human Metal Model compliant waveform with a TLP is described. To characterize this generator, a new analytical method has been developed, which is applicable to both TLP and HMM and demonstrates fundamental differences between those three types of generators. Results are used to correlate failure levels on active devices.

\section{Introduction}

Altogether, the HMM specification [1] and IEC 610004-2 and ISO 10605 standards [2][3] define the most widely used stress pulses in ESD testing and qualification of products. Together, these standards address equipment testing as well as integrated circuit (IC) testing. In case of failure, debugging must be performed to understand the root cause. For integrated circuits in particular, this step can be quite challenging because the pulse waveform is complex and leads to difficult analysis. Often, the simpler waveform of a Transmission Line Pulsing (TLP) system is then employed to try to reproduce the failure. Most of the time, this approach works and a trend can be extracted between a TLP failure current and the ESD gun charging voltage [4]. However, in some cases, this approach won't work and no clear correlation can be established [5]. According to [4], this is particularly the case with $2 \mathrm{k} \Omega$ ESD gun discharge modules. [5] demonstrates that some ESD structures in analog highvoltage technology have completely uncorrelated failure levels between TLP and HMM. Failure analysis shows that the failure mechanisms are different. These few cases are problematic because then TLP testing cannot be wholly trusted to reproduce an ESD gun issue.

In this work, a new setup for generating an HMM compliant waveform with a TLP generator is described. Two additional modules for a standard TLP are created in order to generate an HMM/IEC 61000-
4-2/ISO10605 compliant pulse (Fig. 1) on a coaxial cable. Such approach has been explored successfully in the past by E. Grund [6] and Y. Cao [7]. In [6], a TLP generator is modified by placing an impedance mismatch (resistor) between two coaxial lines. On the other side, in [7], a capacitive discharge through a short coaxial cable is employed to generate an HMMcompliant pulse.

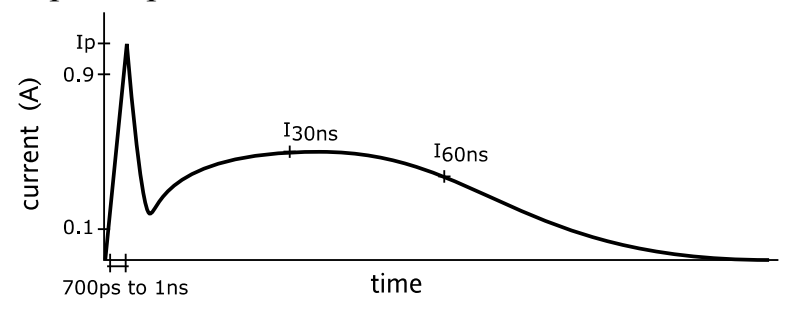

Figure 1: I(t) HMM pulse

The setup described in this paper is a different alternative and reuses a transient effect observed in a previous paper [8] to shape the pulse. The setup is described in Section II. In Section III, passive structures are tested.

The main goal of this paper is developed in Section IV. A simple method is proposed for characterizing the output resistance $R_{O U T}(t)$ (Fig. 2) of ESD generators. $R_{\text {OUT }}(t)$ is an interesting parameter for every ESD generator. For a given load value and charging voltage, the injected current inside the load can be computed easily using Ohm's law in Fig. 2. In this paper, ROUT $(t)$ is characterized for TLP and HMM generators, and the TLP-HMM system described herein. A fundamental 
difference between all three generators is observed and will be detailed.

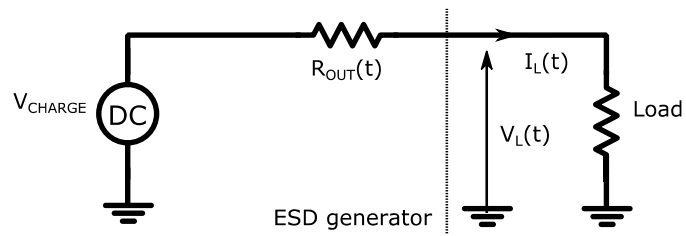

Figure 2: equivalent circuit: ESD generator and load

Finally, this analysis on dynamic output resistance is applied to ESD testing in part V. Failure levels found with HMM, TLP and TLP-HMM are compared. $R_{\text {OUT }}(t)$ is used to build a correlation between those levels. A similar analysis has been conducted previously in [5] on simulations in the frequency domain. However, the method described here to compute $R_{\text {OUT }}(t)$ in the time-domain from simple measurements can be complementary and enables further analysis.

\section{Generator principle}

The new setup described in the paper is composed of two modules that plug directly onto a standard TLP. These modules are simply referred hereafter as "absorber" and "shaping filter" (Fig. 3).

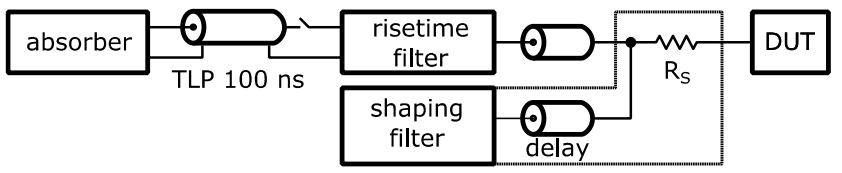

Figure 3: Full TLP-HMM setup.

The shaping filter is made of an assembly of RLC elements. It acts in combination with a delay and a series resistor $R_{S}$. As indicated by its name, it shapes the TLP pulse to match the HMM standard. The absorber located to the left improves the pulse shape by suppressing the pulse reflection of opposite sign. A risetime filter such as described in [9][10] is required to enforce the rise time between $700 \mathrm{ps}$ and $1 \mathrm{~ns}$ as defined in the standards. The HMM pulse (Fig. 1) is comprised of two characteristic parts. It starts with a short peaked pulse section followed by a slower broad pulse section.

\section{Peaked pulse generation}

In Fig. 4, a capacitor $\mathrm{C}$ is separated from the main propagation path by a small transmission line $(\Delta t)$. The inductor $\mathrm{L}$ can be neglected in this first part of the analysis, as it behaves as an open circuit at the beginning of the pulse. The short line introduces a delay $\Delta \mathrm{t}$ between the main line and the RLC elements. When a TLP pulse is injected on the main line, it reaches point $A$ at $t=0$ and the voltage at $A$ rises from $0 \mathrm{~V}$. The capacitor is still "not visible" from A and does not see the TLP rising edge yet.

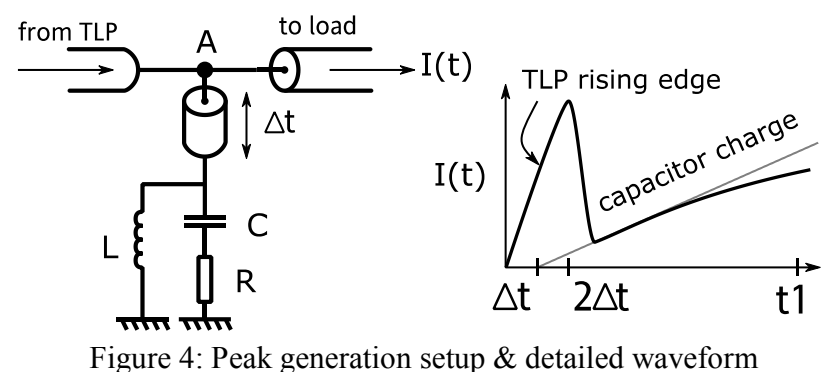

At $t=\Delta t$, the pulse has reached the capacitor which is initially discharged and starts charging. This change in voltage and current in the capacitor branch at $t=\Delta t$ is not visible immediately from point $A$ until it propagates back. Point A keeps rising with the TLP impulse. At $t=2 \Delta t$, the reflection from the capacitor reaches $\mathrm{A}$, whose potential falls almost immediately. This results in the generation of the short first section. The peak width of approximately $2 \Delta t$ on the pulse going to the load. The last element to be explained is the resistor R. It introduces an offset voltage as soon as the capacitor $\mathrm{C}$ starts charging. This offset is used to tune the voltage and (under $1 \Omega$ ) the current $I(t)$ upwards or downwards at $\mathrm{t}=2 \Delta \mathrm{t}$.

\section{Broad pulse generation}

After $2 \Delta t$, point A follows the charging capacitor voltage. If the resistor $\mathrm{R}$ and inductor $\mathrm{L}$ were not connected along with the capacitor, the charge would continue until TLP voltage is reached (with reflections from the short cable neglected). This is where the inductor $\mathrm{L}$ connected in parallel plays a part. At $\mathrm{t}=0$, the inductor is an open circuit and conducts approximately no current. Slowly the current through the inductor increases, and at $\mathrm{t}=\mathrm{t} 1$, it is enough to cancel the capacitor charging current. At this moment, the capacitor voltage increases has stopped and the capacitor starts to discharge through the inductor. Ultimately, the inductor draws all the current and brings the voltage and current on the main line back to 0 . The result of this combined action after $2 \Delta t$ of the capacitor and the inductor leads to the generation of the second part of the pulse.

\section{Shaping filter}

The exact schematic of the shaping filter is given Fig. 5. Capacitances are distributed to reduce parasitic 
inductances. The inductances have also been distributed to increase the maximum total current that can be absorbed.

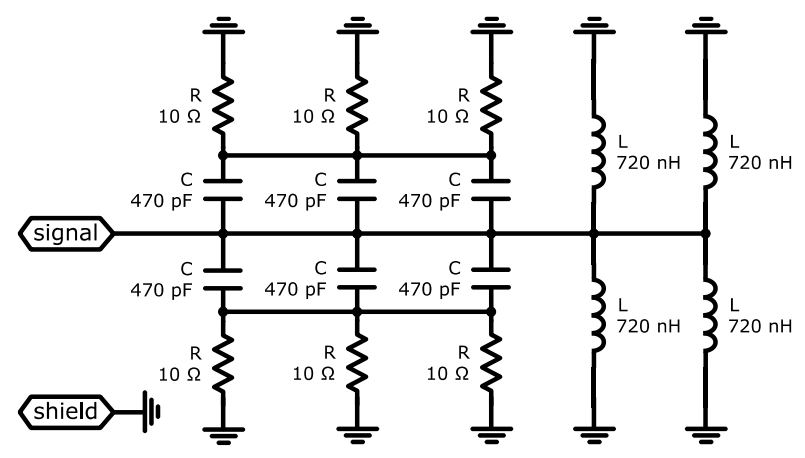

Figure 5: shaping filter schematic

The PCB (Fig. 6) has a ground plane, and the central line is $50 \Omega$ matched. Overall, its dimensions must be kept as small as possible to reduce the impact of delays.

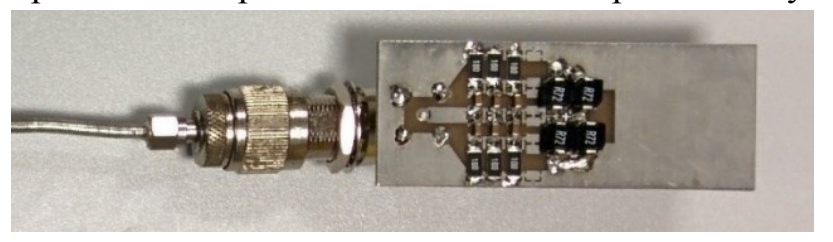

Figure 6: Picture of the shaping filter

In Figure 6, the delay cable $(\Delta t)$ can be seen on the left. As described in II.1, this cable must induce a delay half of the final pulse risetime. To be compliant, the rise time of the pulse must be comprised between 700ps and 1ns. Thus, the delay $\Delta \mathrm{t}$ must be comprised between $350 \mathrm{ps}$ and $500 \mathrm{ps}$ to have a clean peak. In practice, a slightly longer cable ensures the maximum (and desired) peak voltage is reached. The pulse rise time is fixed directly by the TLP rise time, which can be enforced accurately with a rise time filter [9][10].
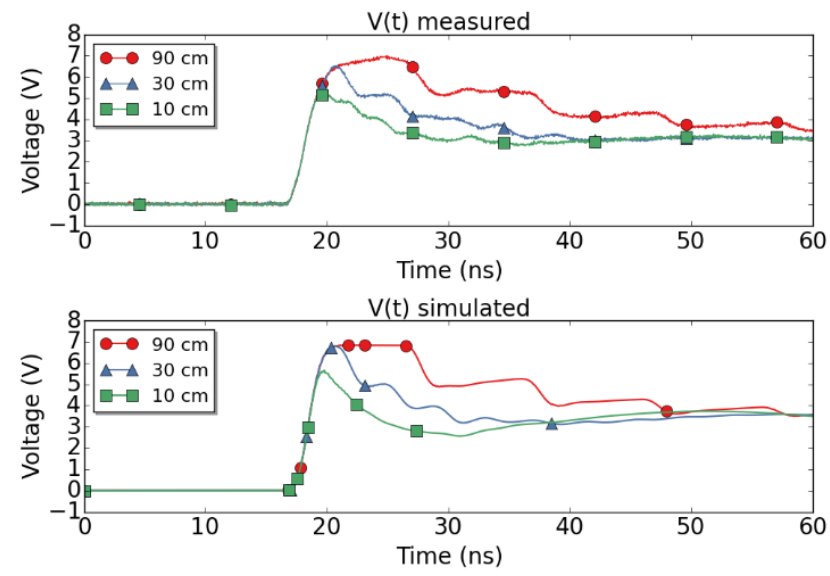

Figure 7: Impact of different cable lengths - V(t) across $2 \Omega$ load measured (top) and simulated (bottom)
Compared to the TLP risetime, a shorter delay for the shaping filter's cable will reduce the amplitude of the peak while a longer delay will let the TLP reach its maximum voltage and generate a short flat region. The impact of different $\Delta \mathrm{t}$ values on the peak is presented on Fig 7. The TLP charging voltage is identical for each curve and the load is $2 \Omega$ (calibration load defined in IEC 61000-4-2). A 2ns rise time filter is employed here for accentuating the interaction between the risetime of the pulse and the delay of the cable. In the final system, a 1 ns risetime filter is used instead to comply with the HMM specification.

The red curve in Fig. 7 corresponds to the shortest cable. The peak amplitude never reaches the maximum voltage. The blue curve corresponds to the optimal length. The peak reaches the maximum voltage and falls immediately. The green curve corresponds to the longest cable. The delay is long enough that the load sees a TLP step for $10 \mathrm{~ns}$ before the voltage falls down. The last element playing a part in the pulse shaping is the series resistor $R_{S}$ (Fig. 3) of value $8 \Omega$. With this resistor, it is easier to match the required ratio between the peak current and the $30 \mathrm{~ns}$ current.

\section{Absorber}

The schematic of the absorber is given Fig. 8. It is constituted of a $50 \Omega$ resistor, in series with $6.6 \mathrm{nF}$. It acts as a matched termination for transient events, and absorbs any incoming reflections.

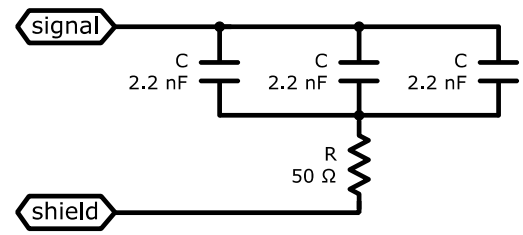

Figure 8: Absorber schematic

Because it is connected to the TLP line but on the opposite side of the load, it will absorb current at the end of the TLP pulse. Thus, the pulse reflection of opposite sign will be globally eliminated by this system. The picture of the absorber is given Fig. 9. It is helpful for understanding a small issue caused by a parasitic capacitance described in Section II.5.

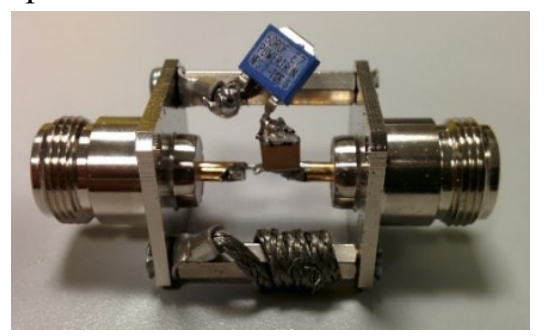

Figure 9: Picture of the absorber 


\section{Compliancy measurements}

The response of the generator is tested in conditions as close as possible to the ISO 10605 standard [3]. Basically, the generator is connected to a $2 \Omega$ load, itself connected to a $12 \mathrm{GHz}$ (10 ps/sample point) oscilloscope with a $50 \Omega$ input impedance. The setup (Fig. 10) is identical to the target measurement setup defined in [2][3] in terms of impedance. The only difference is that the system is entirely connected through coaxial cables.

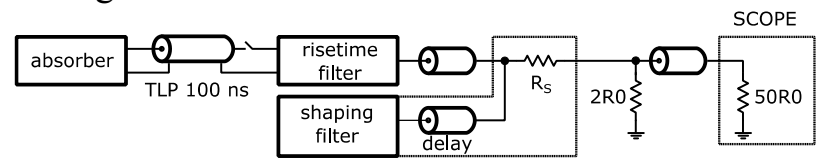

Figure 10: Setup used for compliancy measurement

The resulting waveform is given in Figure 11. The simulation is also provided for comparison.

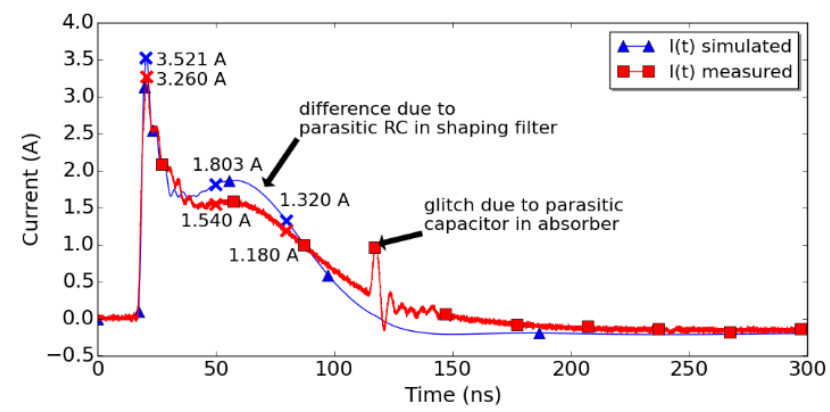

Figure 11: TLP-HMM I(t) under $2 \Omega$ - 250V TLP charging voltage

Table 1: Standard pulse tolerance margins from ISO 10605

\begin{tabular}{|c|c|c|c|c|c|}
\cline { 2 - 7 } \multicolumn{1}{c|}{} & Nominal (A) & $\mathbf{- 1 0 \%}(\mathbf{A})$ & $\mathbf{+ 1 0 \%}$ (A) & simulated (A) & measured (A) \\
\hline Peak & 3.75 & 3.375 & 4.125 & 3.521 & 3.26 \\
\hline & Nominal (A) & $\mathbf{- 3 0 \% ( A )}$ & $\mathbf{+ 3 0 \%}$ (A) & simulated (A) & measured (A) \\
\hline $\mathbf{3 0 ~ n s}$ & 2 & 1.4 & 2.6 & 1.803 & 1.54 \\
\hline $\mathbf{6 0} \mathbf{~ n s}$ & 1 & 0.7 & 1.3 & 1.32 & 1.18 \\
\hline
\end{tabular}

Measured currents at 30ns and 60ns are within the $30 \%$ tolerance of the standard (see Table 1). The measured peak current is a bit lower $(110 \mathrm{~mA}$ short of minimum margin), but this is very easily corrected on the TLP by adding a small positive offset on the charging voltage. However, there is a clear difference from the simulation in the measured signal from $40 \mathrm{~ns}$. This comes from the shaping filter, and the inductances in particular. Their frequency behavior is not as good as expected and having four inductances in parallel has increased this issue. Indeed, in the shaping filter configuration, the parasitic capacitances of inductors are in parallel. Thus, they add together, leading to a degraded frequency behavior. For the next iteration of the shaping filter, a single RF inductor should be used instead.
The shaping filter model can be corrected by connecting in parallel a total parasitic capacitance of $2 \mathrm{nF}$ in series with a $15 \Omega$ parasitic resistor (Fig. 12).

The glitch visible at approximately $120 \mathrm{~ns}$ is due to the absorber, because of two different parasitic devices. At the beginning of the TLP pulse, the parasitic capacitance between signal and ground (estimated to $20 \mathrm{pF}$ in simulation) is charged. Its sudden discharge at the end of the TLP pulse causes the short voltage/current increase observed at $120 \mathrm{~ns}$. Then, the parasitic series inductance of the three $2.2 \mathrm{nF}$ capacitors and the $50 \Omega$ resistor (Fig. 13) are responsible afterward for the small oscillation observed between $120 \mathrm{~ns}$ to $150 \mathrm{~ns}$.

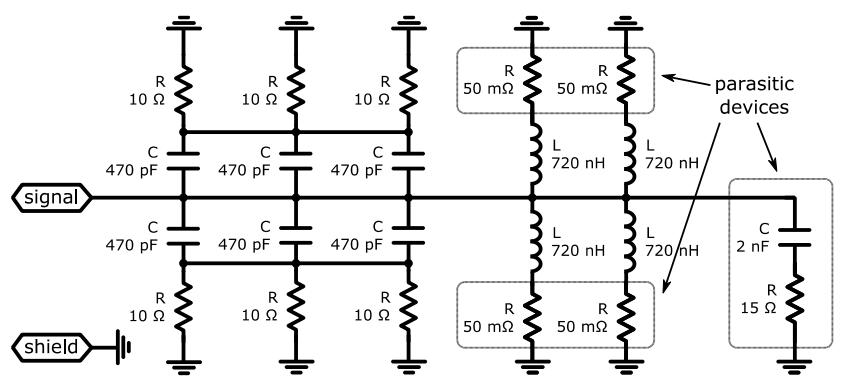

Figure 12: Shaping filter model with parasitic devices

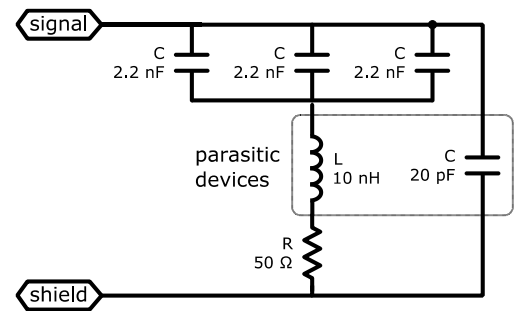

Figure 13: Absorber model with parasitic devices

This issue will be fixed in the next iteration of the absorber by building the absorber on a dedicated PCB with $50 \Omega$ lines. Guarantying matching along the path should eliminate this effect.

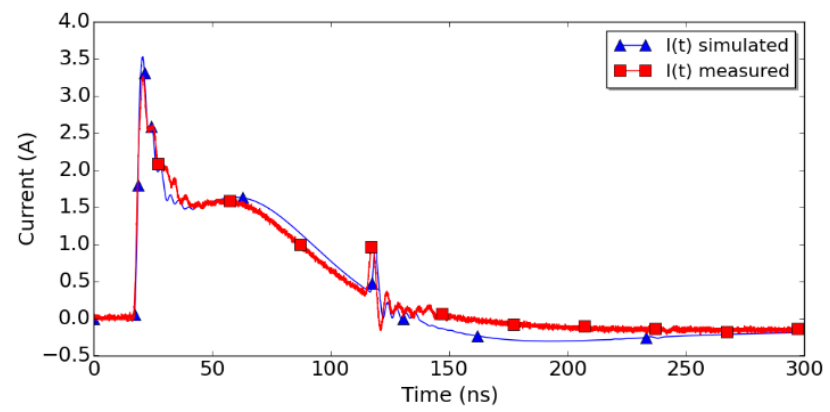

Figure 14: TLP-HMM I(t) under $2 \Omega$ with parasitic devices taken into account- $250 \mathrm{~V}$ TLP charging voltage

The measurement/simulation comparison Fig. 14 takes into account all the parasitic devices in the circuit 
(inductors frequency behavior and absorber parasitic capacitance), leading to a much better correlation.

\section{Resistors testing \& models validation}

In this section, resistive loads of $2 \Omega, 10 \Omega$, and 50 $\Omega$ are tested. The first two loads are simply constituted of several resistors in parallel. The oscilloscope input impedance is used for the $50 \Omega$ load. In this case, reflections are eliminated by the matched load and the waveform is less disturbed. The measurement setup for $2 \Omega$ and $10 \Omega$ is given in Fig. 15 .

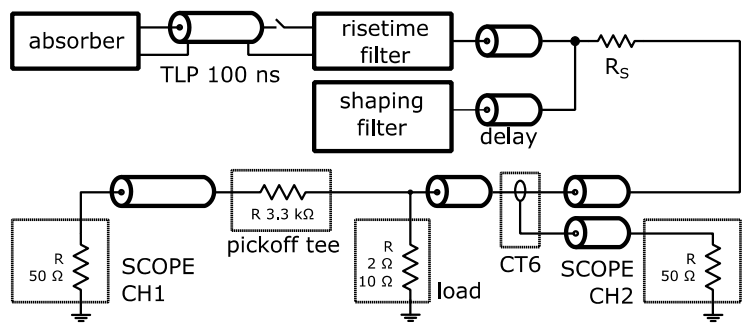

Figure $15: 2 \Omega$ and $10 \Omega$ loads setup
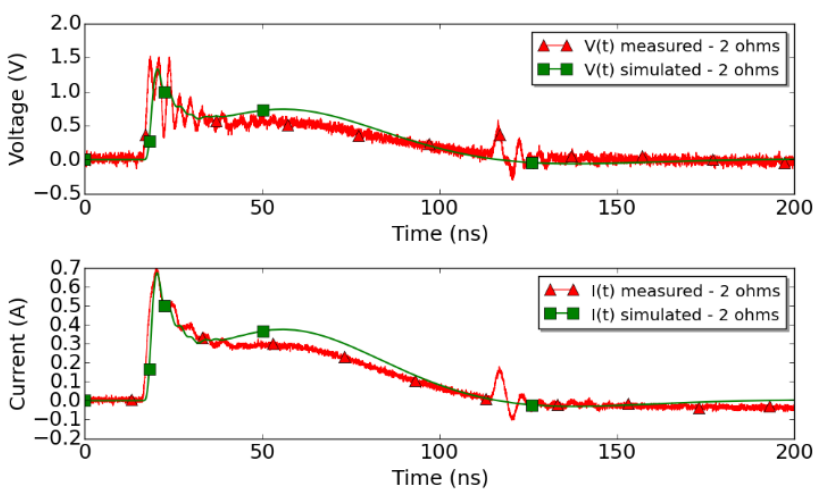

Figure 16: TLP-HMM on $2 \Omega$ load - Measured and simulated V(t)$\mathrm{CH} 1$ (top) and $\mathrm{I}(\mathrm{t})-\mathrm{CH} 2$ (bottom)
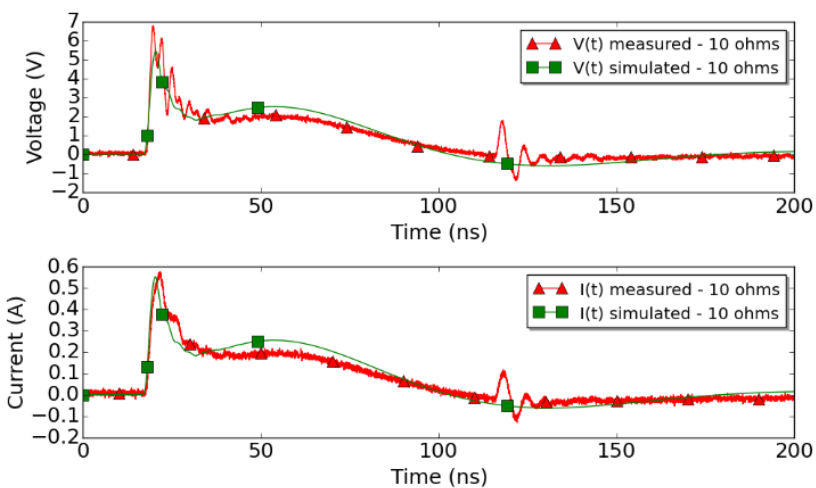

Figure 17: TLP-HMM on $10 \Omega$ load - Measured and simulated $\mathrm{V}(\mathrm{t})-\mathrm{CH} 1$ (top) and $\mathrm{I}(\mathrm{t})-\mathrm{CH} 2$ (bottom)
The oscilloscope has $12 \mathrm{GHz}$ of bandwidth (10 ps/sample point). For $2 \Omega$ and $10 \Omega$, the measurements (Fig. $16 \& 17$ ) are quite noisy because of reflections. Despite that, it is interesting to observe that overall the amplitudes match well. The differences come mostly from parasitic devices not taken into account in those simulation. The setup for the $50 \Omega$ load is much simpler (Fig. 18). A CT6 current transformer is placed between the output of the TLP-HMM and the oscilloscope. A 1:10 attenuator is used to protect the oscilloscope.

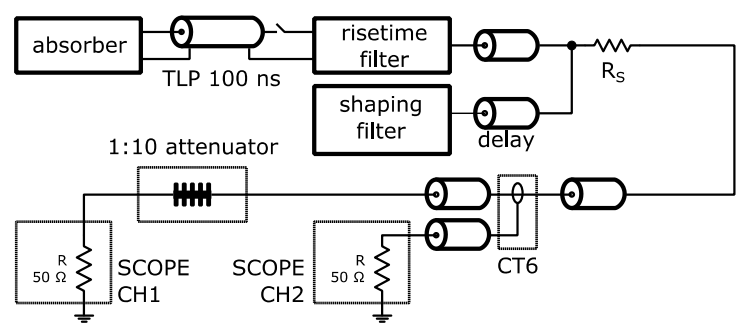

Figure 18: $50 \Omega$ load setup
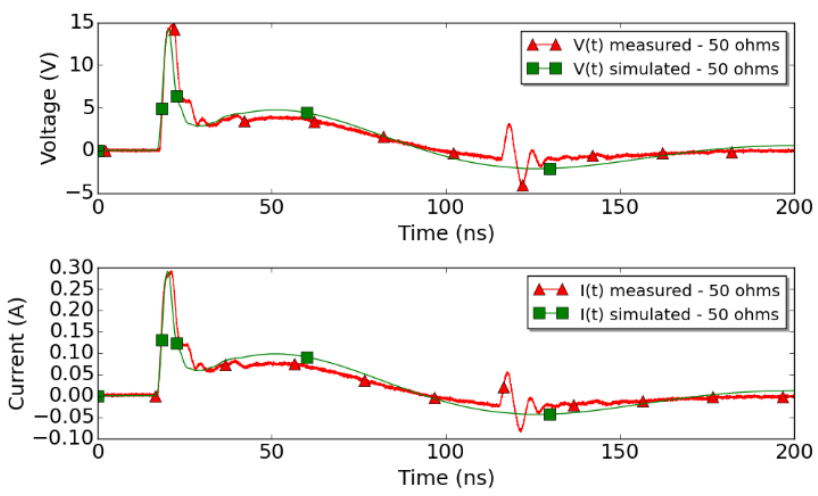

Figure 19: TLP-HMM on $50 \Omega$ load - Measured and simulated $\mathrm{V}(\mathrm{t})-\mathrm{CH} 1$ (top) and $\mathrm{I}(\mathrm{t})-\mathrm{CH} 2$ (bottom)

For a $50 \Omega$ loading, the comparison between simulations and measurements is given Fig. 19. The matched impedance eliminates the reflections and generates less noisy waveforms. Overall, voltage and current match well and the models are considered valid.

\section{Output impedance study \\ 1. Analytic method and model proofing}

The output resistance $R_{\text {OUT }}(t)$ is computed for three different ESD generators (TLP, HMM \& TLP-HMM). To compute it, Equation 1 requires two time-domain waveforms and the charging voltage value:

- $\mathrm{V}_{\text {CHARGE}}$ : Charging voltage

- $\mathrm{V}_{\mathrm{L}}(\mathrm{t})$ : Output voltage on a load

- $\mathrm{I}_{\mathrm{L}}(\mathrm{t})$ : Output current on a load 
Simply by applying Ohm's law in the equivalent circuit given Fig. 20, it is straightforward to compute $R_{\text {OUT }}(t)$ once in possession of $\mathrm{V}_{\text {CHARGE}}, \mathrm{V}_{\mathrm{L}}(\mathrm{t})$ and $\mathrm{I}_{\mathrm{L}}(\mathrm{t})$ (Eq. 1).

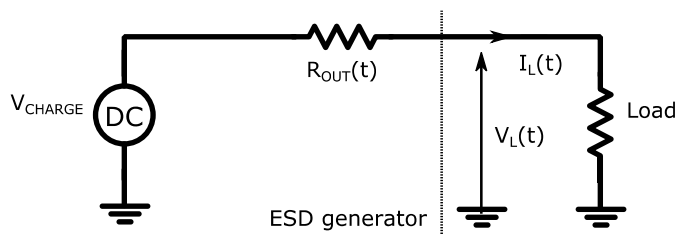

Figure 20: equivalent circuit for ESD generator

$$
\operatorname{Rout}_{\text {OUt }}(t)=\left(V_{\text {CHARGE }}-V_{L}(t)\right) / I_{L}(t)
$$

First, the simulation of $R_{\text {OUT }}(t)$ is checked against measurements with loads of $2 \Omega, 10 \Omega$ and $50 \Omega$ for each generator (Fig. 21 to 23).
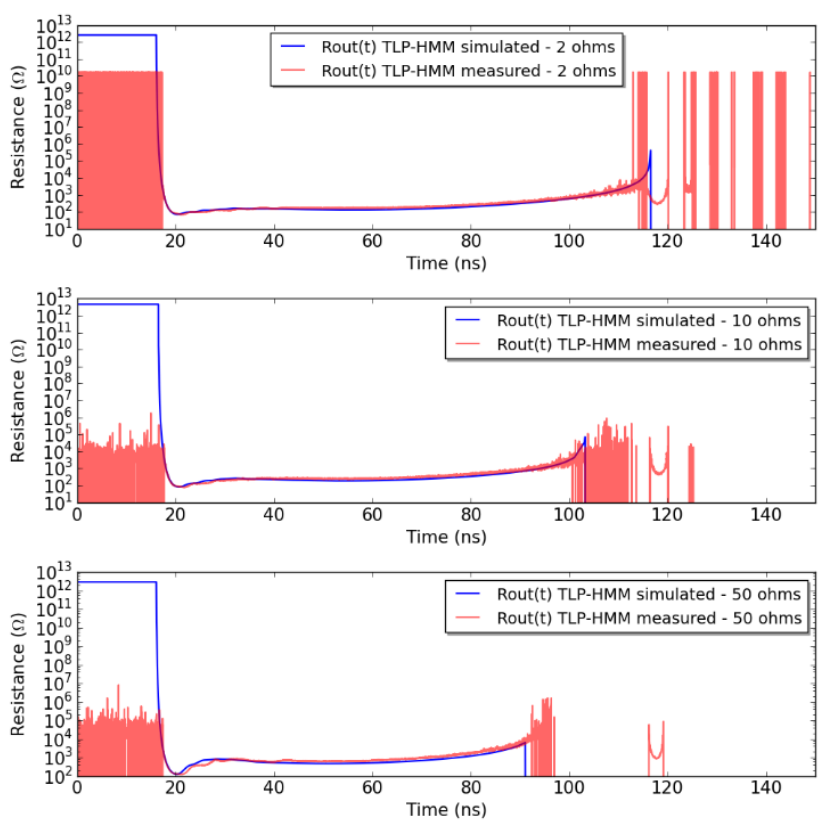

Figure 21: RouT(t) for TLP-HMM - measured (red) \& simulated (blue)

The area of interest is during the first hundred nanoseconds of the ESD pulse, between 20ns and 90ns on the waveforms (the discharge starts at $19 \mathrm{~ns}$ ). In this area, the error on $R_{\text {OUT }}(t)$ for the TLP-HMM model is in the worst case $40 \%$ and is considered acceptable. In Fig. 22, the model is checked for HMM. Overall, the HMM measurements are hardest to perform with low noise. However, the error between simulation and measurements is acceptable. The estimated average output resistance between 20ns and 90 ns is between $1 \mathrm{k} \Omega$ and $700 \Omega$.
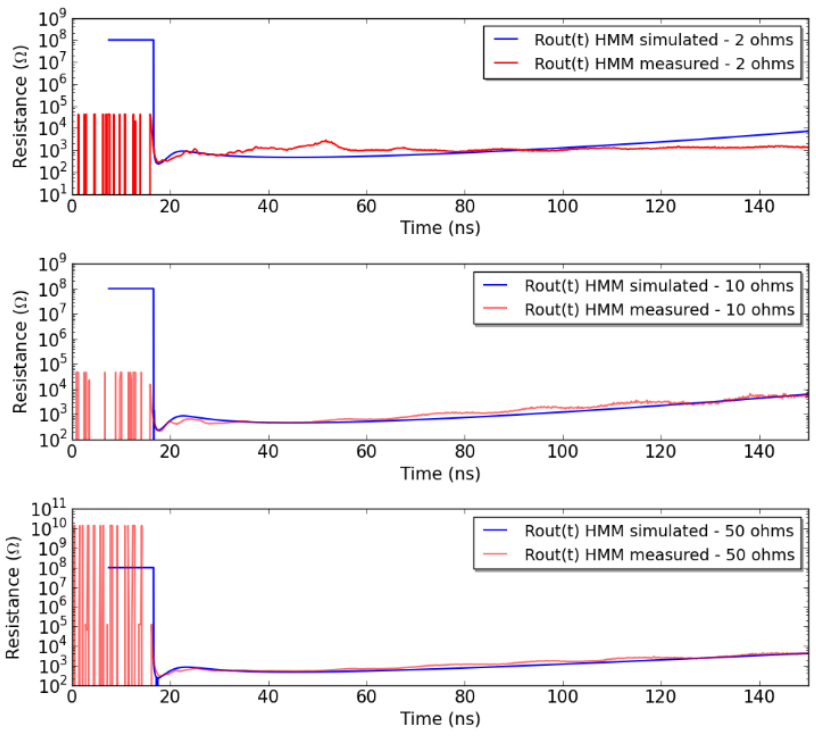

Figure 22: Rout(t) for HMM - measured (red) \& simulated (blue)

Finally, the TLP model is checked (Fig. 23). The average measured resistance between $20 \mathrm{~ns}$ and $90 \mathrm{~ns}$ is slightly higher than expected at $54 \Omega$ instead of $50 \Omega$ in simulation. Otherwise, the TLP simulation closely fits the measurements. There is a difference before $20 \mathrm{~ns}$ (moment where the discharge begins). It is due to the off-resistance of the TLP switch, tuned to $100 \mathrm{M} \Omega$ in the simulation.
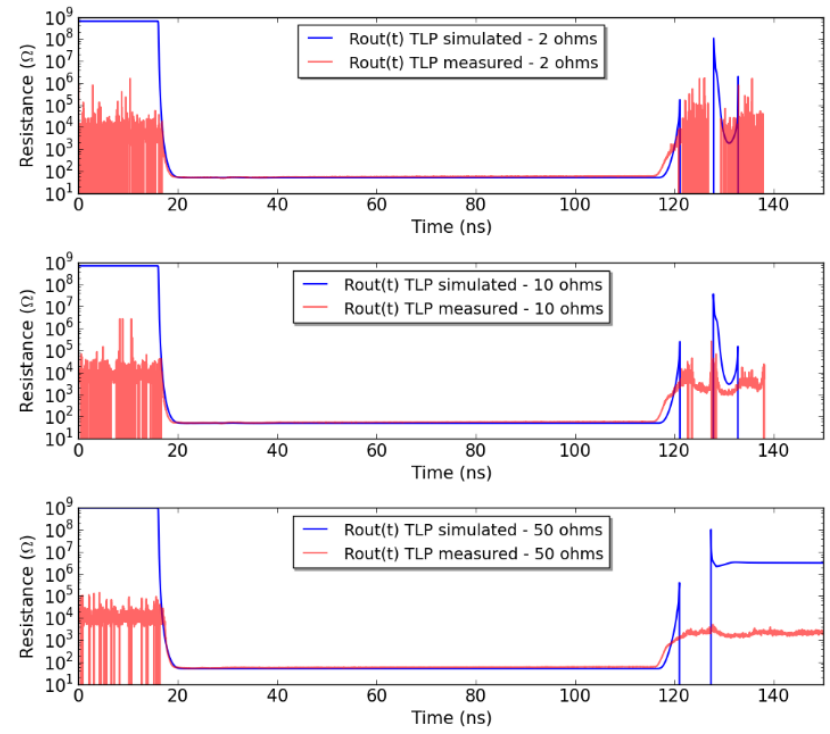

Figure 23: Rout(t) for TLP - measured (red) \& simulated (blue)

In practice, this value is lower but it is very difficult to estimate it from the measurements because of the noise. Indeed, the measured current leaked from the TLP is quite low before the discharge, and thus hard to measure accurately with our setup. This is also true at the end of the discharge (no matter the generator), 
where injected current becomes really small and the measurement error grows quickly.

\section{Curve analysis}

Now that models are proven to work with acceptable tolerance, the study of the different dynamic output resistances is performed only with simulations which are easier to visualize. The impact of the characterization resistor $(2 \Omega, 10 \Omega$ or $50 \Omega)$ is studied for each generator. The TLP, no matter the load, achieves always a constant $50 \Omega$ output resistance, which was expected. The HMM generator also provides a quite stable impedance in the range of studied loads. It is much higher than the TLP, between $500 \Omega$ and $1 \mathrm{k} \Omega$. It is interesting to notice that unlike the TLP, this impedance is not constant in time. It is also higher than $330 \Omega$, which is the value of the gun discharge resistance. The difference between $300 \Omega$ and $500 \Omega-1 \mathrm{k} \Omega$ is most likely due to the discharge capacitor and parasitic inductances that increase the total impedance seen from the output at high frequencies.

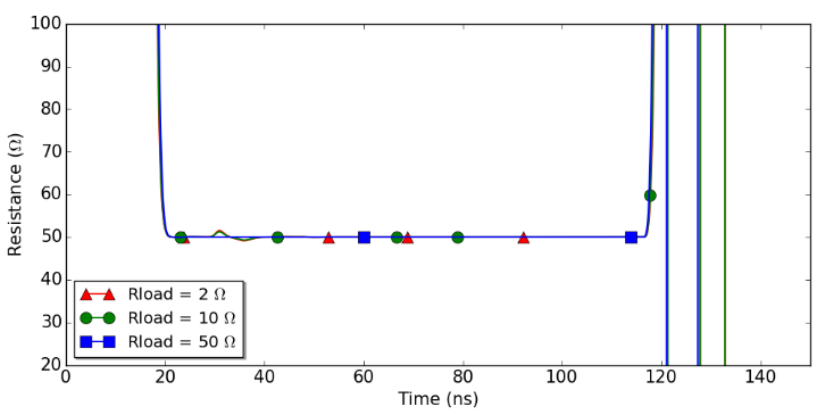

Figure 24: Rout ( $t$ ) for TLP - vertical zoom - simulations only

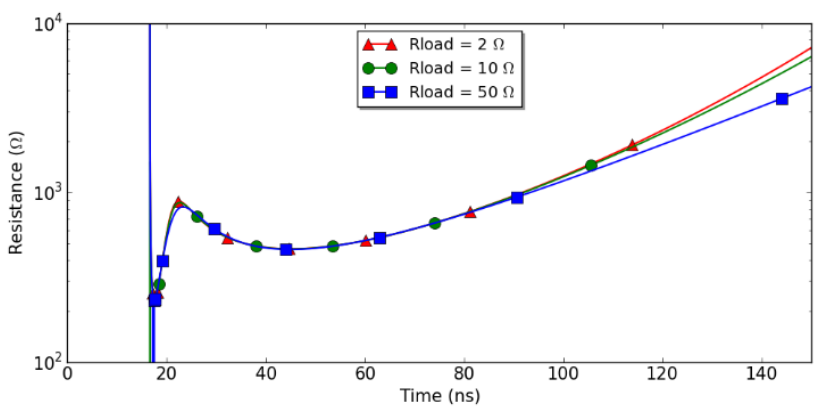

Figure 25: Rout (t) for HMM - vertical zoom - simulations only

Finally, the TLP-HMM is the generator that shows the widest variation. For a $2 \Omega$ load, its output resistance is close to $120 \Omega$ (average between $20 \mathrm{~ns}$ and $90 \mathrm{~ns}$ ) while with a $50 \Omega$ load the output resistance is much higher at
$500 \Omega$. Thus, its output impedance globally evolves between that of the TLP and HMM.

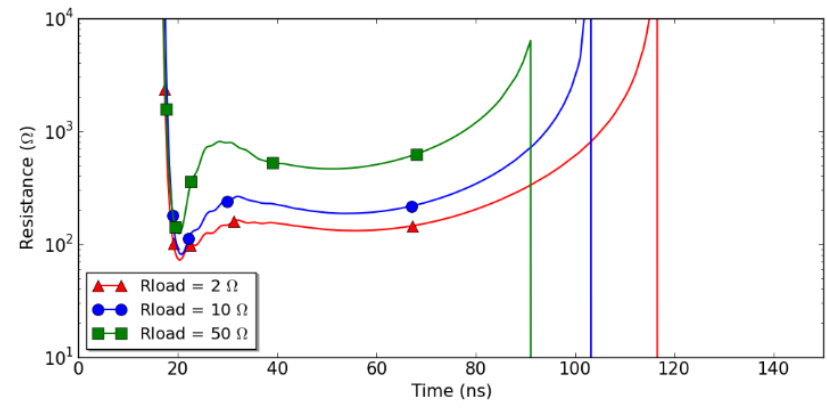

Figure 26: Rout (t) for TLP-HMM - vertical zoom - simulations only

To sum up, these three curves highlight fundamental differences between all the three generators. For both HMM \& TLP-HMM, the output resistance is not constant in time because the discharge paths include capacitors and inductors, which have different current responses as functions of the load. For the TLP-HMM, the current response also depends on the load value for the range of on-resistance usually met with ESD protections. For the TLP, the measured impedance is constant at about $54 \Omega$. Table 2 summarizes the output impedances $\mathrm{R}_{\mathrm{OUT}}$ in function of the load. For the HMM and TLP-HMM, the output resistances Rout provided in this table are simply the average of $R_{O U T}(t)$ between 20ns and 90ns.

Table 2: Output resistance Rout against different loads

\begin{tabular}{|c|c|c|c|c|c|c|}
\hline \multirow[b]{3}{*}{ Load } & \multicolumn{6}{|c|}{ Rout $(\Omega)$} \\
\hline & \multicolumn{2}{|c|}{ TLP } & \multicolumn{2}{|c|}{ TLP-HMM } & \multicolumn{2}{|c|}{ HMM } \\
\hline & measured & simulated & measured & simulated & measured & simulated \\
\hline $2 \Omega$ & \multirow{5}{*}{54} & \multirow{5}{*}{50} & 188 & 123 & 1080 & 607 \\
\hline $5 \Omega$ & & & 1 & 145 & I & I \\
\hline $10 \Omega$ & & & 307 & 179 & 720 & 606 \\
\hline $20 \Omega$ & & & 1 & 254 & 1 & 1 \\
\hline $50 \Omega$ & & & 1060 & 499 & 743 & 600 \\
\hline
\end{tabular}

For the TLP-HMM, it exists a linear relation between the output resistance and the load resistance, as shown in Fig. 27.

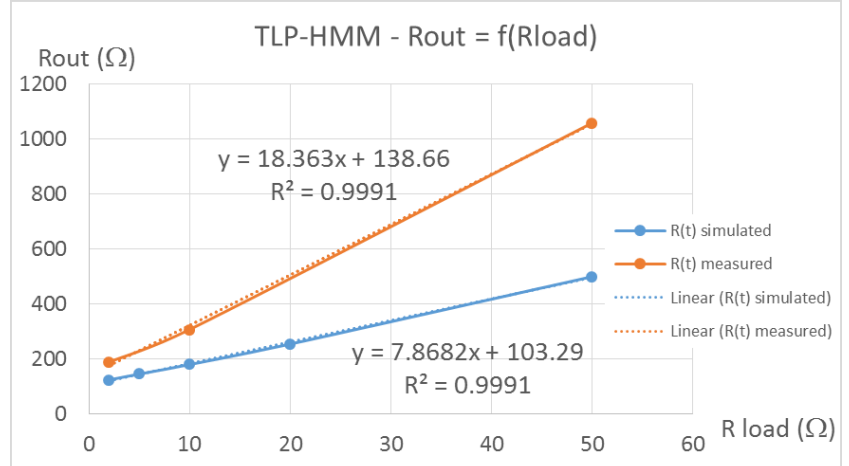

Figure 27: TLP-HMM RouT with different loads - and matching equations 
Equation 2 enables to compute the average output resistance ROUT of the TLP-HMM for a given load:

$$
R_{\text {OUT }}=18.363 * R_{\text {LOAD }}+138.66
$$

In the next section, ESD protections are tested and RouT is used to build a failure correlation between generators based on failure currents.

\section{ESD Protections testing}

\section{Testing}

The differences between all three test generators have been quantified. Now, 4 different ESD protections are tested until breakdown. The goal is see if a mathematical relation can be established between failures levels found with each generator, using their dynamic output resistance computed in Section IV.

Table 3: Table for RON of each ESD structure

\begin{tabular}{|c|c|}
\hline Structure & RoN (ohms) \\
\hline structure A & 6.2 \\
\hline structure B & 2.85 \\
\hline structure C & 9.72 \\
\hline structure D & 13.3 \\
\hline
\end{tabular}

The test procedure is standard and consists in monitoring the leakage current after each new pulse. Failure levels are compared for TLP, TLP-HMM and HMM. Five samples are tested for each structure with each generator to ensure that the failure levels do not have large variations in failure levels. This number of samples is still quite low, but is considered sufficient for this study. The results are summarized in Table 4 .

Table 4: Average failure levels with TLP, HMM \& TLP-HMM

\begin{tabular}{|c|c|c|c|c|}
\hline Structure & $\begin{array}{c}\text { TLP failure } \\
\text { current (A) }\end{array}$ & $\begin{array}{c}\text { TLP failure } \\
\text { charging } \\
\text { voltage (V) }\end{array}$ & $\begin{array}{c}\text { TLP-HMM failure } \\
\text { charging voltage } \\
\text { (V) }\end{array}$ & $\begin{array}{c}\text { HMM failure } \\
\text { charging voltage } \\
\text { (kV) }\end{array}$ \\
\hline A & $2.15(+/-0.15)$ & $142(+/-8)$ & $640(+10)$ & 1.25 \\
\hline B & $2.2(+-0.1)$ & $147(+/-4)$ & $700(+10 /-20)$ & $1.25(-0.25)$ \\
\hline C & $2.23(+-0.1)$ & $168(+/-4)$ & $890(+10 /-20)$ & $1.5(+0.25)$ \\
\hline$D$ & $2.135(+-0.1)$ & $175(+/-5)$ & $860(+20 /-10)$ & $1.5(-0.25)$ \\
\hline
\end{tabular}

The structures used in this study fail at rather small current (about $2 \mathrm{~A}$ ). This is due to hardware limitations imposed by the TLP bench on the charging voltage, and thus on the maximum current deliverable by the TLPHMM. Also, the fact that the output impedance of the TLP-HMM increases with the load accentuates this issue. This means that with this generator, the injected current is lower than expected for ESD protections with on-resistance above $2 \Omega$. Clearly, the generator proposed in this paper is a study tool rather than an alternative to mainstream ESD generators.

\section{Comparison of failure currents}

To establish a correlation between the failure levels found with each generator, three parameters are employed:

- Output resistance $\mathrm{R}_{\text {OUT }}$ of the generator (computed in Section IV)

- On-resistance $\mathrm{R}_{\mathrm{ON}}$ of the ESD protection (Table 3)

- Charging voltages $V_{\text {CHARGE }}$ at failure (Table 4)

Using these three parameters, a very simple approach is used. With the equivalent circuit (Fig. 28), the failure currents are computed for each generator using the following formula:

$$
\mathbf{I}_{\text {ESD }}=\mathbf{V}_{\text {CHARGE }} /\left(\mathbf{R}_{\text {OUT }}+\mathbf{R}_{\text {ON }}\right)
$$

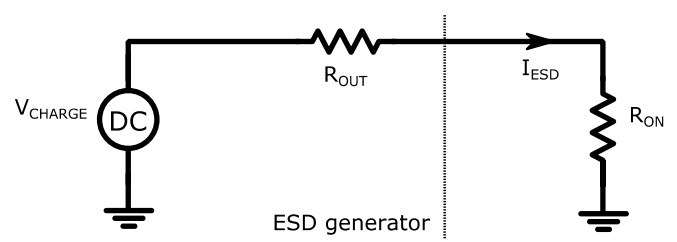

Figure 28: equivalent circuit with the ESD generator and the ESD protection

For TLP-HMM, the output resistance $\mathrm{R}_{\mathrm{OUT}}$ depends on the load. To compensate for this variation, Equation 2 is used with $\mathrm{R}_{\mathrm{LOAD}}$ taken equal to the ESD protection's on-resistance. Tables 5 to 7 summarize the failure current computed with Equation 3.

Table 5: IESD computation for TLP-HMM

\begin{tabular}{|c|c|c|c|}
\hline Structure & V charge TLP-HMM & TLP-HMM Rout & IESD (A) TLP-HMM \\
\hline A & 644 & 253 & 2.5 \\
\hline B & 700 & 192 & 3.6 \\
\hline C & 886 & 316 & 2.7 \\
\hline D & 862 & 380 & 2.2 \\
\hline
\end{tabular}

Table 6: IESD computation for TLP

\begin{tabular}{|c|c|c|c|}
\hline Structure & V charge TLP & TLP Rout & IESD (A) TLP \\
\hline A & 142 & 53 & 2.4 \\
\hline B & 147 & 53 & 2.6 \\
\hline C & 168 & 53 & 2.7 \\
\hline D & 175 & 53 & 2.6 \\
\hline
\end{tabular}

Table 7: IESD computation for HMM

\begin{tabular}{|c|c|c|c|}
\hline Structure & V charge HMM & HMM Rout & IESD (A) HMM \\
\hline A & 1250 & 605 & 2.0 \\
\hline$B$ & 1250 & 607 & 2.0 \\
\hline C & 1500 & 600 & 2.5 \\
\hline$D$ & 1500 & 600 & 2.4 \\
\hline
\end{tabular}

All computed failure currents are compared in Fig. 29. The values are quite close independently of the generator. This tends to show that a correlation exists 
between the failing levels found for each generator (for these 4 different structures).

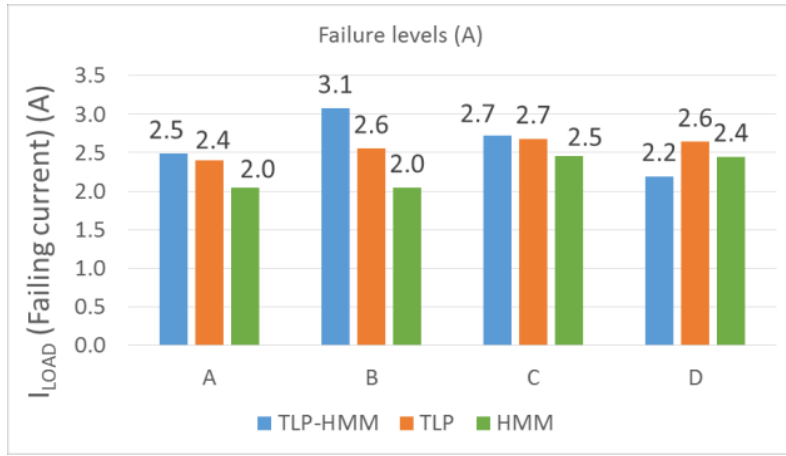

Figure 29: Comparison of computed failure currents

Therefore, in the conditions of this test, the correlation law is simply that the $\mathrm{I}_{\mathrm{ESD}}$ failure current seems constant independently of the test generator. To verify further this relation, more structures with higher current capabilities are tested in the next section.

\section{Analysis of stronger ESD structures}

Because of the limitations on the TLP-HMM charging voltage, the structures presented in this part will only

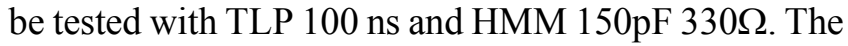
goal is to validate the correlation law on more structures, with failure currents much higher than 2A. For these measurements, a different TLP generator is used than in the other parts of the article. It has a $50 \Omega$ resistor between signal and ground after the relay for absorbing reflections (Fig. 30). In terms of dynamic output resistance, a part of the incoming current is absorbed and for a given charging voltage, the output current is lower. Thus, the output resistance $\mathrm{R}_{\text {OUT }}$ is a bit higher (simulated at $83 \Omega$ in average between $20 \mathrm{~ns}$ and $90 \mathrm{~ns}$ ). This TLP was not built for the purpose of this paper and its configuration could not be changed. It is however interesting to notice that some tricky TLP configurations can lead to output impedances quite different of $50 \Omega$.

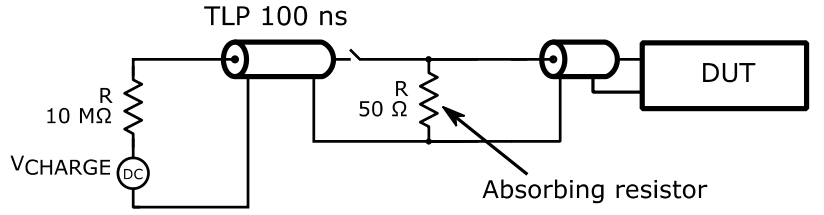

Figure 30: TLP configuration - used during high current structures characterization

The ESD protections $\mathrm{E}$ to $\mathrm{J}$ are presented in Table 8.
Table 8: RON for high current structures

\begin{tabular}{|c|c|}
\hline Structure & RoN (ohms) \\
\hline E & 2.7 \\
\hline F & 3.25 \\
\hline G & 2.42 \\
\hline H & 4.7 \\
\hline I & 1.68 \\
\hline J & 1.86 \\
\hline
\end{tabular}

Once again, Equation 3 is used with TLP and HMM to compute the failure current $\mathrm{I}_{\mathrm{ESD}}$. The results are summarized in Tables $9 \& 10$ and a comparison is provided in Fig. 31.

Table 9: IESD for HMM

\begin{tabular}{|c|c|c|c|}
\hline Structure & V charge HMM & HMM Rout & IESD (A) HMM \\
\hline E & 6500 & 605 & 10.7 \\
\hline F & 5000 & 607 & 8.2 \\
\hline G & 13000 & 600 & 21.6 \\
\hline H & 9500 & 600 & 15.7 \\
\hline I & 20000 & 600 & 33.2 \\
\hline J & 14500 & 600 & 24.1 \\
\hline
\end{tabular}

Table 10: IESD for TLP

\begin{tabular}{|c|c|c|c|}
\hline Structure & V charge TLP & TLP Rout & IESD (A) TLP \\
\hline E & 870 & 83 & 10.2 \\
\hline F & 710 & 83 & 8.2 \\
\hline G & 1630 & 83 & 19.1 \\
\hline H & 1200 & 83 & 13.7 \\
\hline I & 2630 & 83 & 31.1 \\
\hline J & 1860 & 83 & 21.9 \\
\hline
\end{tabular}

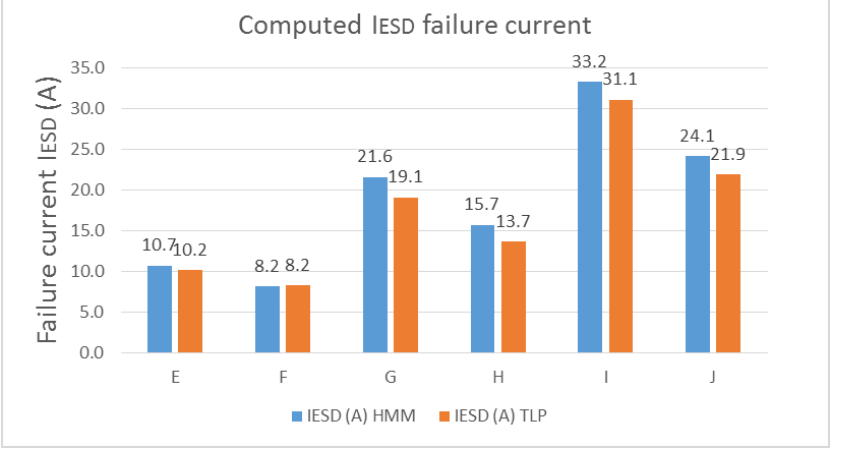

Figure 31: Comparison of IESD computed failure currents with HMM (blue) and TLP (orange) using our correlation method

Similarly as in Section V.2., values for $\mathrm{I}_{\mathrm{ESD}}$ are very close between TLP and HMM. The correlation method seems to work well in the given test conditions. Now, in the case where only the TLP charging voltage and on-resistance of the ESD structure are known, it is possible to compute the current $\mathrm{I}_{\mathrm{ESD}}$ at which the structure will fail. IESD is then used in equation 4, which is once again ohm's law applied to the equivalent circuit (Fig. 32). This equation computes the HMM charging voltage that induces a current IESD,TLP (obtained from TLP measurement) into a protection 
ESD of resistance $\mathrm{R}_{\mathrm{ON}}$ (computed from same TLP measurement).

$$
\mathbf{V}_{\text {HMM }}=\mathbf{I}_{\text {ESD.TLP }} *\left(\mathbf{R}_{\text {OUT,HMM }}+\mathbf{R}_{\text {ON }}\right)
$$

Equation 4 is applied to structures A to F. For each structure, the predicted HMM voltage from TLP measurements is compared to the actual one. Results are summarized in Fig 32.

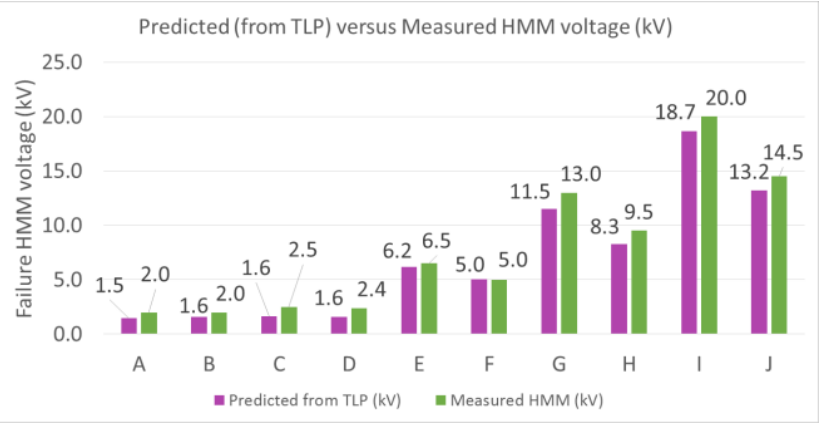

Figure 32: Comparison of predicted \& actual HMM failure charging voltage

The correlation method works well in this case and predicts the HMM charging voltage at which the device will fail from the TLP measurement with decent accuracy. To validate this approach even further, it would be necessary to perform the analysis with the other ESD gun discharge modules, and to test a larger set of ESD protections.

\section{Conclusion}

A technique for shaping an HMM pulse on a 50 ohms line was described in this paper. The waveform generated on a $2 \Omega$ resistor is compliant with the HMM specification.

A new method for characterizing ESD generators was presented, which allows computing the dynamic output resistance of any ESD generator. The initial motivation for this method was to quantify the differences between the TLP-HMM and more standard stress generators such as TLP and HMM.

ESD protection structures were tested with those three generators. The output resistance of each generator, combined with the on-resistance resistance of the ESD protection, enables to compute the failing current of the devices. On a set of 10 different ESD structures, it turned out that a correlation law could be established, meaning that the HMM failure level could be predicted from a TLP characterization of the device. The validity range of this method remains to be verified on a much larger set of ESD protections, especially in the cases where the failure mechanism is suspected to not be a thermal breakdown.

\section{References}

[1] "Human Metal Model" specification, technical report, ESD Association, Working Group 5.6

[2] IEC 61000-4-2. Electromagnetic compatibility (EMC) - Part 4-2: testing and measurement techniques - electrostatic discharge immunity test. Ed 2.0, 2008-12. ISBN: 2-8318-1019-7.

[3] ISO10605 standard, "Road vehicles - Test methods for electrical disturbances from electrostatic discharge"

[4] Patrice Besse, Jean-Philippe Laine, Alain Salles, Mike Baird, "Correlation between System Level and TLP Tests Applied to Stand-alone ESD Protections and Commercial Products", (EOS/ESD), 2010

[5] M. Scholz, S.-H. Chen, D. Johnsson et. Al., "Miscorrelation between IEC61000-4-2 type of HMM tester and 50 ohms HMM tester", Electrical Overstress/Electrostatic Discharge Symposium (EOS/ESD), 2012

[6] Evan Grund, Kathleen Muhonen, Nathaniel Peachey, "Delivering IEC 61000-4-2 Current Pulses through Transmission Lines at 100 and 330 ohm System Impedances", Electrical Overstress/Electrostatic Discharge Symposium (EOS ESD), 2008

[7] Yiqun Cao, Werner Simburger, David Johnsson, "A TLP-based Human Metal Model ESDgenerator for device qualification according to IEC 61000-4-2", Asia-Pacific International Symposium on Electromagnetic Compatibility, April 12 - 16, 2010, Beijing, China

[9] Rémi Bèges, Fabrice Caignet et. Al., "Practical Transient System-level ESD ModelingEnvironment Contribution", Electrical Overstress/Electrostatic Discharge Symposium EOS ESD, 2014

[10] Djordjevic et al., "On a Class of Low-Reflection Transmission-Line Quasi-Gaussian Low-Pass Filters and Their Lumped-Element Approximations", in IEEE Trans. on Microwave Theory and Techniques, July 2003.

[11] Yiqun Cao, Werner Simbuirger, David Johnsson, "Rise-Time Filter Design for Transmission line Pulse Measurement Systems", Microwave Conference, March 2009, Germany 